\title{
Single-institute prospective trial of laparoscopy-assisted distal gastrectomy with systemic lymph node dissection for early gastric carcinoma
}

\author{
Yoshinari Mochizuki • Yasuhiro Kodera • \\ Michitaka Fujiwara $\cdot$ Yuichi Ito $\cdot$ Kazunari Misawa \\ Yukihide Kanemitsu $\cdot$ Seiji Ito
}

Received: 27 December 2010/Accepted: 15 July 2011/Published online: 13 August 2011

(C) The International Gastric Cancer Association and The Japanese Gastric Cancer Association 2011

\begin{abstract}
Background Laparoscopy-assisted gastrectomy (LAG) is an advanced surgery that requires the mastery of complex surgical skills. We evaluate the feasibility of LAG with systemic lymph node dissection when participating surgeons have sufficient knowledge and experience to conduct open surgery for gastric cancer and basic laparoscopic skills.

Methods All operations were performed by two Japan Surgical Society board-certified attending surgeons who had performed over 50 conventional gastrectomies and 30 laparoscopic cholecystectomies. The surgeons went through an established program, including training at the wet and dry laboratories. In addition, surgeries for the first 10 cases were assisted by an expert surgeon with experience of $>300$ cases. To be eligible for the LAG procedure, patients had to have a preoperative diagnosis of T1, N0 and M0 gastric carcinoma. The morbidity rate was used as the study endpoint. Variables such as operating time, intraoperative blood loss and number of retrieved lymph nodes were evaluated as complementary surgical endpoints. These variables were compared between the first 25 cases and the latter 25 cases.
\end{abstract}

\footnotetext{
Y. Mochizuki · Y. Ito · K. Misawa · Y. Kanemitsu · S. Ito Department of Gastroenterological Surgery,

Aichi Cancer Center Hospital, Nagoya, Japan

Y. Kodera $(\bowtie) \cdot$ M. Fujiwara

Department of Surgery II, Nagoya University School

of Medicine, 65 Tsurumai-cho, Showa-ku,

Nagoya 466-8550, Japan

e-mail: ykodera@med.nagoya-u.ac.jp
}

Results A total of 50 patients who were scheduled to undergo LAG were prospectively enrolled between 2005 and 2008. Morbidity rate was $4 \%$ (2/50), with one case due to intestinal injury and one case due to an intra-abdominal abscess. Complications related to laparoscopy were observed in $2 \%$ (1/50), with one case of mesenteric injury. The conversion rate to laparotomy was $6 \%$ (3/50). However, there were no serious consequences in converted cases. The operating time was $263.7 \pm 45.0 \mathrm{~min}$. The intraoperative blood loss was $94.5 \pm 106.5 \mathrm{~g}$. The total number of regional lymph nodes retrieved was $34.7 \pm 12.2$. A significant improvement in the blood loss was only noted after the first 25 procedures. All patients are alive and disease-free after a median follow-up of 38.8 months.

Conclusion An adequate training program, including site visits by expert surgeons, in conjunction with basic laparoscopy skills and solid backgrounds in open gastrectomy from the perspective of the trainees are currently key to the successful and safe implementation of LAG. Whether the procedure is oncologically feasible remains to be confirmed by long-term follow-up.

Keywords Gastric carcinoma - Laparoscopy-assisted gastrectomy · Prospective trial · Surgical training · Surgical background

\section{Introduction}

In the past decade, laparoscopic techniques have gained wide clinical acceptance in surgical practice. The important advantages offered by this approach, as compared to the open surgery procedure, include reduced intraoperative blood loss, reduced postoperative pain and accelerated recovery, earlier return to normal bowel function with 
earlier resumption of oral intake, and earlier discharge from the hospital. These potential benefits observed for laparoscopic cholecystectomy have prompted surgeons to expand this approach to other procedures, including the resection of neoplastic diseases. Laparoscopic surgery is considered the criterion standard for the treatment of several benign diseases and some malignant diseases such as colon cancer [1]. However, laparoscopic-assisted gastrectomy (LAG) for gastric cancer has yet to be validated, for several reasons. First, LAG with systemic lymph node dissection is technically quite difficult to perform. In addition, LAG has only been performed by a limited number of surgeons dedicated to laparoscopic surgery. Finally, few reports have described the advantages of performing LAG for early gastric cancer, and most of these were retrospective case series of a limited number of patients. Thus, LAG is not applicable as a standard treatment for gastric cancer. At the present time, the Japanese Gastric Cancer Association (JGCA) states that LAG should be performed only in a clinical trial setting in accordance with their gastric cancer treatment guidelines [2].

Since laparoscopic surgery has been proven to have substantial advantages over conventional surgery, it is foreseeable that LAG will gain significance in the future and will eventually be regarded as a standard of care. As far as it goes, LAG requires abilities well beyond the basic technique and knowledge of laparoscopic surgery in order to conduct clinical trials safely. In the current study, we prospectively evaluated our experience of implementing this procedure in a high-volume hospital, which involved adhering to a strict training program and working under the guidance of an established expert in this field, while maintaining basic ethical principles. Our aim was to show that the technique used to perform LAG can be acquired safely under an adequate instruction and training program; it is not possible at this time to show that this surgery is oncologically equivalent to the open surgery.

\section{Patients and methods}

\section{Patients}

Between August 2005 and March 2008, we enrolled 51 consecutive patients for LAG with lymph node dissection. Demographic, clinical, operative, and pathologic data were collected. Eligibility criteria were as follows: performance status of ECOG 0-1; age range between 20 and 75 years old; histologically proven gastric adenocarcinoma; location of the primary tumor within the lower and middle third of the stomach; presence of $\mathrm{cT} 1, \mathrm{cN} 0$ and $\mathrm{cM} 0$ as diagnosed by endoscopy, barium study and CT; sufficient organ function; and written informed consent.
Surgeons

To be considered capable of performing LAG, surgeons were required to be board-certified by the Japan Surgical Society, to have performed at least 50 open gastrectomies with lymph node dissection, and to have completed at least 30 laparoscopic cholecystectomies. In addition, surgeons had to be trained in the Nagoya University Endoscopic Surgery (NUESS) training laboratory and animal laboratory in accordance with the Nagoya University School of Medicine, Surgery II Educational Program for LAG. This educational program consisted of three components: (1) suturing training in the box trainer; (2) virtual reality (VR) simulator training using MIST (SimSurgery, AS, Oslo, Norway), the Lap Sim System (Surgical Science Ltd, Gothenburg, Sweden), and Lap Mentor (Simbionix Corporation, Cleveland, OH, USA), and; (3) a hands-on training course for LAG in a porcine model. Of the three VR simulators, MIST was mainly used to improve suturing skills. The Lap Sim System consists of 11 different exercises featuring camera navigation, instrument navigation, hand coordination, grasping, cutting, clip applying, lifting and grasping, suturing, precision and speed, handling intestines, and fine dissection, and was used to teach a wide range of basic skills. Lap Mentor offers training in complete laparoscopic surgical procedures through virtual reality laparoscopic cholecystectomy. We were required to participate in the NU-ESS training scheme twice within 1 year before starting the study.

\section{Procedures}

Two board-certified surgeons (Y.M. and S.I.) were chosen to operate on the trial patients. Ten initial operations were supervised by an experienced mentor who had acquired the Endoscopic Surgical Skill Qualification and has performed $>300$ laparoscopy-assisted gastrectomies (M.F.). All operations after the first 10 were performed by either of the two surgeons, while the other surgeon invariably took part as an assistant. A total of 6 surgical residents who rotated the group for 6 months during the study period handled the video scope. These residents also had experience of at least 10 open gastrectomies as either surgeon or assistant. LAG was performed according to a previously published approach [3]. Lymph node dissection and ligation of vessels were carried out in the laparoscopic field. Lymphadenectomy was performed according to the JGCA guidelines. Laparoscopyassisted pylorus-preserving gastrectomy (LAPPG) was only indicated for mucosal carcinoma located in the middle third, while laparoscopy-assisted distal gastrectomy (LADG) was used in the remaining early carcinoma cases. The gastrectomy and subsequent gastroduodenostomy used the same methodology employed in open surgery, with the procedure performed through a 4-cm midline skin incision window. 


\section{Endpoints}

Morbidity rate was used as the study endpoint. We hypothesized that the lower limit of the $95 \%$ CI for the observed morbidity rate would not exceed the historical control morbidity rate of $11.6 \%$, which is the rate that was calculated for the 1153 cases of open distal gastrectomy with systemic lymph node dissection performed between 1994 and 2003 at our institute.

Other perioperative outcomes, including operating time, intraoperative blood loss, conversion rates, and retrieved lymph nodes, were evaluated and also adopted as learning indicators. The learning curve was defined as the comparison of the first 25 procedures with the second 25 .

Ethics

Written informed consent was obtained from all patients participating in the study.

The study protocol was approved by the Institutional Review Board of the Aichi Cancer Center Hospital. The trial was overseen by an independent data monitoring committee.

\section{Results}

Clinicopathological characteristics of the patients

A total of 50 patients (31 men and 19 women; mean age \pm standard deviation, $57.4 \pm 10.8$; mean body mass index, $21.9 \pm 2.9$ ) who were scheduled to undergo LAG were prospectively enrolled between 2005 and 2008. Three of these 50 patients had undergone a previous surgery. Details of the surgical procedures and the pathologic data are presented in Table 1.

\section{Surgical outcomes}

The mean operating time was $263.7 \pm 45.0 \mathrm{~min}$. The average amount of blood loss during the surgery was $94.5 \pm 106.5 \mathrm{~mL}$. None of the patients required any transfusions during or after the operations. The total number of regional lymph nodes retrieved was $34.7 \pm 12.2$ (range 30-46).

A complication related to the laparoscopy occurred in one case and included injury of the transverse colon mesentery. The situation was resolved intra-operatively without any hardship or further critical complications. In another case, there was a postoperative complication involving an intra-abdominal abscess on the anterior surface of the head of the pancreas, which was treated conservatively. The overall morbidity rate was $4 \%(2 / 50)$. The lower limit of the
Table 1 Surgical procedures and pathological data

\begin{tabular}{|c|c|}
\hline Variables & No. of cases \\
\hline \multicolumn{2}{|l|}{ Surgical procedure } \\
\hline LADG & 40 \\
\hline LAPPG & 8 \\
\hline LADG + cholecystectomy $^{\mathrm{a}}$ & 2 \\
\hline \multicolumn{2}{|l|}{ Lymph node dissection } \\
\hline D1 + alpha & 16 \\
\hline D1 + beta & 34 \\
\hline \multicolumn{2}{|l|}{ Tumor location } \\
\hline Middle third & $38^{\mathrm{b}}$ \\
\hline Lower third & $18^{\mathrm{b}}$ \\
\hline Lower-middle third & $1^{\mathrm{b}}$ \\
\hline \multicolumn{2}{|l|}{ No. of primary tumors } \\
\hline Solitary & 43 \\
\hline Multiple & 7 \\
\hline \multicolumn{2}{|l|}{ Depth of invasion } \\
\hline T1 (mucosal) & 28 \\
\hline T2 (submucosal) & 20 \\
\hline T3 (muscle propria) & 2 \\
\hline \multicolumn{2}{|l|}{ Lymph node metastasis } \\
\hline No & 47 \\
\hline N1 & 1 \\
\hline $\mathrm{N} 2$ & 2 \\
\hline \multicolumn{2}{|l|}{ Distant metastasis } \\
\hline M0 & 50 \\
\hline M1 & 0 \\
\hline \multicolumn{2}{|l|}{ Stage } \\
\hline IA & 46 \\
\hline IB & 2 \\
\hline II & 1 \\
\hline IIIA & 1 \\
\hline
\end{tabular}

${ }^{a}$ Contributed to cholecystectomy for co-existing cholelithiasis

b Number of lesions

95\% CI for this morbidity rate did not exceed the historical control morbidity rate. A total of three $(6 \%)$ out of the 50 cases required conversion to laparotomy due to uncontrollable bleeding $(n=1)$, injury of the transverse colon mesentery $(n=1)$ or other disease at the lesser curvature of the remnant stomach, which turned out to be suspicious for gastrointestinal stromal tumor by frozen section $(n=1)$. All three cases were restored by open surgery, and none of the patients developed any postoperative complications. There was no difference in operating time, blood loss, and the incidence of postoperative complications between these patients and those whose surgeries were completed by the laparoscopy-assisted approach. There were no deaths related to the laparoscopic surgery; nor were there any operative or in-hospital deaths. All patients are alive and disease-free after a median follow-up of 38.8 months. 
Comparison of the first 25 procedures

with the second 25

A comparison of the perioperative outcomes of the first 25 procedures with the second 25 is shown in Table 2. No significant difference was observed with the exception of the mean estimated blood loss, which decreased significantly from 125.8 to $63.2 \mathrm{~g}$. No trend towards shorter operating time can be seen in Fig. 1.

\section{Discussion}

Laparoscopic cancer surgeries that require advanced skills are now considered to be an acceptable alternative to open surgery in patients with colorectal cancer [1]. Prospective randomized studies have demonstrated that both short- and long-term outcomes after laparoscopic resection for colorectal cancer are comparable to those observed for the open approach [4-6]. This has led the American Society of Colon and Rectal Surgery to issue a statement approving the procedure [7]. However, laparoscopic surgery has yet to

Table 2 Comparison of postoperative outcomes between first 25 cases and second 25 cases

\begin{tabular}{llll}
\hline & First 25 cases & $\begin{array}{l}\text { Second } \\
25 \text { cases }\end{array}$ & $P$ \\
\hline Ope. time (min, mean \pm SD) & $268.4 \pm 45.1$ & $258.9 \pm 45.4$ & NS \\
Blood loss (g, mean \pm SD) & $125.8 \pm 113.6$ & $63.2 \pm 90.6$ & 0.03 \\
No. of LN (no., mean \pm SD) & $35.0 \pm 9.2$ & $34.4 \pm 14.8$ & NS \\
Morbidity (\%) & $1(4)$ & $1(4)$ & NS \\
Mortality (\%) & 0 & 0 & NS \\
Conversion (\%) & $2(8)$ & $1(4)$ & NS
\end{tabular}

Ope. time operating time, blood loss intra-operative blood loss, no. of $L N$ number of retrieved lymph nodes

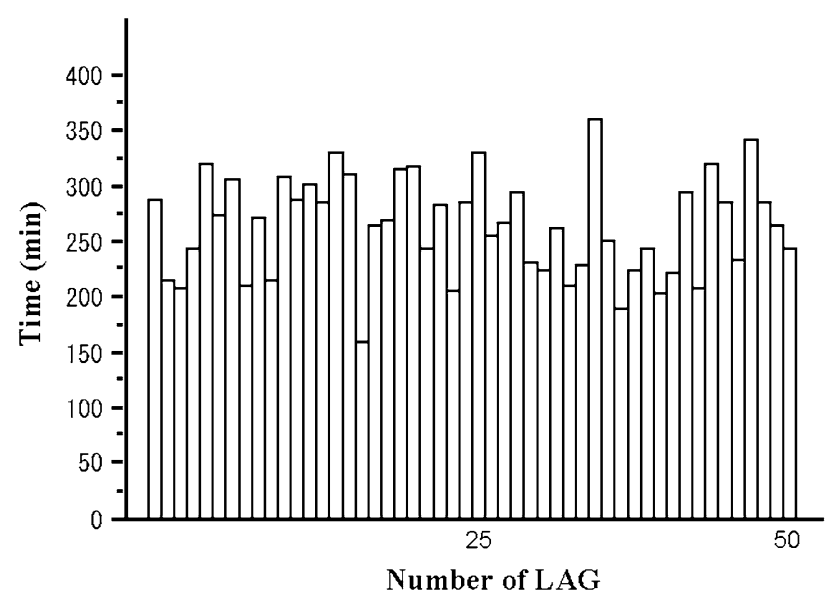

Fig. 1 Operating time for LAG be validated for gastric cancer and is thus only performed in a limited number of gastric cancer patients. In addition, many of these previous studies were retrospective, and therefore, while they are informative, the conclusions drawn can only be viewed as preliminary. In addition, LAG with lymphadenectomy is a complex procedure and requires not only advanced laparoscopic skills but also skills in performing open gastrectomy procedures. As a result, the JGCA currently does not endorse LAG in a curative cancer resection setting outside of clinical trials due to concerns over potentially compromised cancer control [2].

However, because of substantial advantages such as less pain, more rapid recovery of bowel function, and better cosmetic results, LAG has rapidly gained popularity for gastric cancer in Japan. We believe that a solid background in both gastrectomy and basic laparoscopic technique is required before surgeons can ethically and safely perform LAG.

Several previous reports have examined the relationship between board certification and surgical outcome. Prystowsky et al. [8] reported that American Board of Surgery certification was associated with reduced mortality and morbidity for colorectal resection. Pearce et al. [9] found that surgeons with a subspecialty certification had better outcomes for carotid endarterectomy and abdominal aortic aneurysm repair. More interestingly, Avital et al. [10] reported that adequate oncologic resections in laparoscopic treatment might be achieved earlier, provided that surgeons adhere to open standard cancer resection methods. Ryu et al. [11] also indicated that the learning period for LAG could decrease when participating surgeons had substantial experience of open gastric surgery. Parikh et al. [12] provided data on the learning curve for open gastrectomy with lymphadenectomy, and postulated that the operator should have performed more than 25 of these procedures under supervision before being considered to be competent in this technique. We assumed, therefore, that experience of 50 cases as an operator would be sufficient for the surgeon to have acquired sufficient technical skills to perform open gastric cancer surgery. Since both of the surgeons that participated in the current study were not only board-certified by the Japan Surgical Society but they also had more than sufficient experience in open gastrectomy, they were defined here as having a solid background in gastric cancer surgery.

Since LAG is a very demanding procedure from a technical point of view, adequate training in laparoscopic techniques and methodology is mandatory. Following training in wet and dry laboratories, several studies have demonstrated improved performance with a significant learning curve [13-16]. In the current study, the surgeons, in accordance with the Educational Program for LAG at 
Table 3 Comparison of outcomes in case series of LAG

\begin{tabular}{|c|c|c|c|c|c|c|c|c|c|}
\hline Year & Author & Pros./retro. & No. & $\begin{array}{l}\text { Ope. time } \\
\text { (min) }\end{array}$ & Blood loss $(\mathrm{g})$ & No. of LN & Mortality (\%) & Morbidity (\%) & $\begin{array}{l}\text { Conversion } \\
(\%)\end{array}$ \\
\hline 2000 & Shimizu [18] & Retro. & 21 & $299 \pm 61$ & $273 \pm 200$ & $14 \pm 10$ & 0 & 19 & 4.7 \\
\hline 2002 & Kitano [19] & Pros. & 14 & $227 \pm 7$ & $117 \pm 30$ & $20 \pm 4$ & 0 & 14 & 0 \\
\hline 2003 & Fujiwara [3] & Pros. & 43 & $225 \pm 56$ & $239 \pm 322$ & $20.2 \pm 9.9$ & 0 & 16.2 & 2.3 \\
\hline 2005 & Hayashi [20] & Pros. & 14 & $378 \pm 97$ & $327 \pm 245$ & $28 \pm 14$ & 0 & 0 & 0 \\
\hline 2005 & Huscher [21] & Pros. & 30 & $196 \pm 21$ & $229 \pm 144$ & $30 \pm 14.9$ & 3.3 & 23.3 & - \\
\hline 2006 & Ziqiang [22] & Retro & 44 & $255 \pm 46$ & $147 \pm 109$ & $30.1 \pm 16.9$ & 0 & 13.6 & 2.2 \\
\hline 2007 & Lee [23] & Pros. & 64 & 280 & 196 & 50.1 & 0 & 3.1 & 6.6 \\
\hline 2008 & Ryu [11] & Retro. & 347 & $270 \pm 59$ & - & - & 0 & 9.8 & 2.3 \\
\hline 2009 & Our study & Pros. & 50 & $263.7 \pm 45.0$ & $94.5 \pm 106.5$ & $34.7 \pm 12.2$ & 0 & 4 & 6 \\
\hline
\end{tabular}

$L A G$ laparoscopy-assisted pylorus-preserving gastrectomy and laparoscopy-assisted distal gastrectomy, pros. prospective study, retro. retrospective study, no. number of patients, ope. time operating time, blood loss intra-operative blood loss, no. of $L N$. number of retrieved lymph node

Department of Surgery II, went through an intensive training course consisting of simulator training and at least three sessions of hands-on training with animals, and obtained basic skills by performing laparoscopic cholecystectomies. Although there is currently no strong evidence to indicate that performing cholecystectomies laparoscopically prior to proceeding to more advanced surgery is beneficial, it is reasonable to assume that the skills acquired during one laparoscopic procedure are transferable to another laparoscopic procedure. Since the experience of 30 laparoscopic cholecystectomies was required to achieve optimum proficiency in basic laparoscopic surgery [17], participating surgeons in the current study were required to have performed at least 30 laparoscopic cholecystectomies. Such a program was not available when earlier attempts to implement LAG were made, and a series reported by our instructing surgeon (M.F.) has revealed a high incidence of leakage (14\%) and greater blood loss $(239 \mathrm{~mL})$ in his initial 43 patients [3]. Our results implied that skills acquired in these training sessions were actually transferable to the operative setting. As for the dry laboratory training mandated in the current study, the box trainer and VR simulators were used to achieve basic laparoscopic surgical competence and were not specific for LAG. Some of the components were not considered compulsory for surgeons who already had sufficient experience with laparoscopic cholecystectomy. Nevertheless, suturing technique is considered a mandatory skill needed to acquire the Endoscopic Surgical Skill Qualification organized by the Japan Society for Endoscopic Surgery, an ultimate goal of the surgeons who participated in this study. The two surgeons (Y.M. and S.I.) trained in the current study successfully acquired the Endoscopic Surgical Skill Qualification as of 2011, and participation in the dry laboratory training was helpful in that aspect.

Concerning the oncologic safety of the laparoscopic approach to gastric carcinoma, the mean number of lymph nodes retrieved from resected specimens was compatible with several series of LAG (Table 3), and fulfilled the minimal number required to evaluate $\mathrm{pN}$ in the TNM classification [24]. Furthermore, no port-site metastasis or recurrent disease was observed after a median follow-up period of 38.8 months. These findings, along with other studies, suggest that the laparoscopic approach is oncologically feasible for the treatment of early gastric carcinoma. Further evidence in this aspect will have to wait for the results of randomized phase III trials; we were allowed to participate in those trials due to the efforts described in the current study.

Since laparoscopic surgery for gastric cancer is considered a technically complex procedure, the learning curve period could be prolonged. Several previous reports have suggested that LAG with lymph-node dissection may require at least 50-90 cases for a surgeon to gain competence [25-27]. It was reported that complications frequently occur during the introductory period, where the greatest of precaution must be taken $[3,28]$. In the current study, blood loss was the only parameter that showed a significant difference between the first and last 25 cases. In other words, the operating time did not significantly shorten during the same period. The operating time in the current series is comparable to those for other reported series (Table 3), however, so it may be that the plateau was attained more rapidly. More importantly, the current study clearly showed that surgeons who were trained adequately in open gastrectomy were capable of achieving acceptable short-term outcomes, even during the initial phase, if sufficient preparation was performed and instructions from a surgeon with the Endoscopic Surgical Skill Qualification were available.

There have been several studies from established laparoscopic surgeons comparing the short-term outcome of LAG with that of conventional open gastrectomy. In a randomized trial, Huscher et al. [21] reported mortality and 
morbidity rates of 3.3 and $26.7 \%$ for LAG, which were equivalent to those for open gastrectomy. Other studies have also reported that morbidity rates associated with LAG are as low as those found for conventional open gastrectomies [29, 30]. The mortality and morbidity rates in the current study were 0 and $4 \%$, respectively, and less than those observed in our historical series of open surgeries. They were at least comparable with those in a series of LAGs reported from other expert institutions (Table 3), demonstrating the adequacy of our method of implementing the new technique. We have had only one major surgical complication, an intra-abdominal abscess, but this was also handled conservatively.

We encountered one other intra-operative complication that was peculiar to the laparoscopic approach: a mesenteric injury due to disorientation resulting in a partial colectomy. However, by converting to open surgery, we were able to successfully complete the gastrectomy, and there were no further complications. In general, higher complication rates have been noted in patients that required conversions during laparoscopic cholecystectomy, appendectomy and colectomy as compared to the open approach [31-34]. However, Slim et al. [35] found that while the conversion generally resulted in an inferior outcome compared to that reported for open colorectal surgery, none of the patients who underwent a conversion at an early stage experienced a complicated postoperative course. Their findings conclusively demonstrate that an early decision to abandon the laparoscopic approach is the key to avoiding serious adverse events. Though our conversion rate was $6 \%$ and was higher than that observed in previous reports (Table 3), there were no serious consequences in our converted cases. Unnecessarily prolonged operating time was also avoided, as shown by the lack of a significant difference between the laparoscopy-only and conversion groups. Adequately judged and timely abandonment of the laparoscopic approach could therefore be considered part of a well-conducted surgery rather than a surgical failure.

To conclude, an adequate training program, including site visits by expert surgeons, in conjunction with established basic laparoscopy skills and a solid background in open gastrectomy from the perspective of the trainees are currently tkey to successfully and safely implementing LAG. It is not possible at this time to show whether LAG is oncologically feasible. The results of phase III trials comparing open surgery and LAG for early-stage gastric cancer by the Korean and Japanese study groups will ultimately answer this issue.

Acknowledgments We have not received any funds for the study.

\section{References}

1. Clinical Outcomes of Surgical Therapy Study Group. A comparison of laparoscopically assisted and open colectomy for colon cancer. N Engl J Med. 2004;350:2050-9.

2. Sano T. In: Japanese Gastric Cancer Society, editors. Guideline for Diagnosis and Treatment of Carcinoma of the Stomach, October 2010 edition. Tokyo: Kanehara; 2010.

3. Fujiwara M, Kodera Y, Kasai Y, Kanyama Y, Hibi K, Ito K, et al. Laparoscopy-assisted distal gastrectomy with systemic lymph node dissection for early gastric carcinoma. J Am Coll Surg. 2003;196:75-81.

4. Braga M, Vignali A, Gianotti L, Zuliani W, Radaelli G, Gruarin P, et al. Laparoscopic versus open colorectal surgery: a randomized trial on short-term outcome. Ann Surg. 2002;236:759-67.

5. Lacy AM, Delgado S, Castells A, Prins HA, Arroyo V, Ibarzabal A, et al. The long-term results of a randomized clinical trial of laparoscopy-assisted versus open surgery for colon cancer. Ann Surg. 2008;248:1-7.

6. Hewett PJ, Allardyce RA, Bagshaw PF, Frampton CM, Frizelle FA, Rieger NA, et al. Short-term outcomes of the Australasian randomized clinical study comparing laparoscopic and conventional open surgical treatments for colon cancer: the ALCCaS trial. Ann Surg. 2008;248:728-38.

7. The American Society of Colon and Rectal Surgeons. Approved statement: laparoscopic colectomy for curable cancer. Surg Endosc. 2004;18:A1.

8. Prystowsky JB, Bordage G, Feinglass JM. Patient outcomes for segmental colon resection according to surgeon's training, certification, and experience. Surgery. 2002;132:663-72.

9. Pearce WH, Parker MA, Freinglass J, Ujiki M, Manheim LM. The importance of surgeon volume and training in outcomes for vascular surgical procedures. J Vasc Surg. 1999;29:768-76.

10. Avital S, Hermon H, Greenberg R, Karin E, Skornick Y. Learning curve in laparoscopic colorectal surgery: our first 100 patients. Isr Med Assoc J. 2006;8:683-6.

11. Ryu KW, Kim YW, Lee JH, Nam BH, Kook MC, Choi IJ, et al. Surgical complications and the risk factors of laparoscopyassisted distal gastrectomy in early gastric cancer. Ann Surg Oncol. 2008; 15:1625-31.

12. Parikh D, Johnson M, Chagla L, Lowe D, McCulloch P. D2 gastrectomy: lessons from a prospective audit of the learning curve. Br J Surg. 1996;83:1595-9.

13. Sturm LP, Windsor JA, Cosman PH, Cregan P, Hewett PJ, Maddern GJ. A systemic review of skills transfer after surgical simulator training. Ann Surg. 2008;4:166-79.

14. Aggarwal R, Ward J, Balasundaram I, Sains P, Athanasiou T, Darzi A. Proving the effectiveness of virtual reality simulation for training in laparoscopic surgery. Ann Surg. 2007;246:771-9.

15. Grantcharov TP, Kristiansen VB, Bendix J, Bardram L, Rosenberg J, Funch-Jensen P. Randomized clinical trial of virtual reality simulation for laparoscopic skills training. Br J Surg. 2004;91:146-50.

16. Olinger A, Pistorius G, Lindemann W, Vollmar B, Hildebrandt U, Menger MD. Effectiveness of a hands-on training course for laparoscopic spine surgery in a porcine model. Surg Endosc. 1999;13:118-22.

17. Moore MJ, Bennett CL. The learning curve for laparoscopic cholecystectomy. The Southern Surgeons Club. Am J Surg. 1995;170:55-9.

18. Shimizu S, Uchiyama A, Mizumoto K, Morisaki T, Nakamura K, Shimura $\mathrm{H}$, et al. Laparoscopically assisted distal gastrectomy for early gastric cancer: is it superior to open surgery? Surg Endosc. $2000 ; 14: 27-31$. 
19. Kitano S, Shiraishi N, Fujii K, Yasuda K, Inomata M, Adachi Y. A randomized controlled trial comparing open vs laparoscopyassisted distal gastrectomy for the treatment of early gastric cancer: an interim report. Surgery. 2002;131:306-11.

20. Hayashi H, Ochiai T, Shimada H, Gunji Y. Prospective randomized study of open laparoscopy-assisted distal gastrectomy with extraperigastric lymph node dissection for early gastric cancer. Surg Endosc. 2005;19:1172-6.

21. Huscher CG, Mingoli A, Sgarzini G, Sansonetti A, Di Paola M, Recher A, et al. Laparoscopic versus open subtotal gastrectomy for distal gastric cancer: five-year results of a randomized prospective trial. Ann Surg. 2005;241:232-7.

22. Ziqiang W, Feng Q, Zhimin C, Miao W, Lian Q, Huaxing L, et al. Comparison of laparoscopically assisted and open radical gastrectomy with extended lymphadenectomy for gastric cancer. Surg Endosc. 2006;20:1738-43.

23. Lee JH, Kim YW, Ryu KW, Lee JR, Kim CG, Choi IJ, et al. A phase-II clinical trial of laparoscopy-assisted distal gastrectomy with D2 lymph node dissection for gastric cancer patients. Ann Surg Oncol. 2007;14:3148-53.

24. Sobin LH, Wittekind CH. TNM classification of malignant tumors. 5th ed. Heidelberg: Springer; 1997.

25. Kim MC, Jung GJ, Kim HH. Learning curve of laparoscopyassisted distal gastrectomy with systemic lymphadenectomy for early gastric cancer. World J Gastroenterol. 2005;11:7508-11.

26. Kunisaki C, Makino H, Yamamoto N, Sato T, Oshima T, Nagano $\mathrm{Y}$, et al. Learning curve for laparoscopy-assisted distal gastrectomy with regional lymph node dissection for early gastric cancer. Surg Laparosc Endosc Percutan Tech. 2008;18:236-41.
27. Zhang X, Tanigawa N. Learning curve for laparoscopic surgery for gastric cancer, a laparoscopic distal gastrectomy-based analysis. Surg Endosc. 2009;23:1259-64.

28. Yoo CH, Kim HO, Hwang SI, Son BH, Shin JH, Kim H. Shortterm outcomes of laparoscopic-assisted distal gastrectomy for gastric cancer during a surgeon's learning curve period. Surg Endosc. 2009;23:2250-7.

29. Pugliese R, Maggioni D, Sansonna F, Scandroglio I, Ferrari GC, Di Lernia S, et al. Total and subtotal laparoscopic gastrectomy for adenocarcinoma. Surg Endosc. 2007;21:21-7.

30. Lee JH, Yom CK, Han HS. Comparison of long-term outcomes of laparoscopy-assisted and open distal gastrectomy for early gastric cancer. Surg Endosc. 2009;23:1759-63.

31. Lo CM, Fan ST, Liu CL, Lai EC, Wong J. Early decision for conversion of laparoscopic to open cholecystectomy for treatment of acute cholecystitis. Am J Surg. 1997;173:513-7.

32. Hellberg A, Rudberg C, Enochsson L, Gudbjartson T, Wenner J, Kullman E, et al. Conversion from laparoscopic to open appendectomy: a possible drawback of the laparoscopic technique? Eur J Surg. 2001;167:209-13.

33. Falk PM, Beart RW Jr, Wexner SD, Thorson AG, Jagelman DG, Lavery IC, et al. Laparoscopic colectomy: a critical appraisal. Dis Colon Rectum. 1993;36:28-34.

34. Hoffman GC, Baker JW, Fitchett CW, Vansant JH. Laparoscopic-assisted colectomy. Initial experience. Ann Surg. 1994; 219:732-43.

35. Slim K, Pezet D, Riff Y, Clark E, Chipponi J. High morbidity rate after converted laparoscopic colorectal surgery. Br J Surg. 1995; $82: 1406-8$ 